\title{
Space-borne polarimetric SAR sensors or the golden age of radar polarimetry
}

\author{
E. Pottier \\ IETR, UMR CNRS- 6164, Université de Rennes 1, 35042, Rennes, France
}

\begin{abstract}
SAR Polarimetry represents an active area of research in Active Earth Remote Sensing. This interest is clearly supported by the fact that nowadays there exists, or there will exist in a very next future, a non negligible quantity of launched Polarimetric SAR Spaceborne sensors. The ENVISAT satellite, developed by ESA, was launched on March 2002, and was the first Spaceborne sensor offering an innovative dualpolarization Advanced Synthetic Aperture Radar (ASAR) system operating at C-band. The second Polarimetric Spaceborne sensor is ALOS, a Japanese Earth-Observation satellite, developed by JAXA and was launched in January 2006. This mission includes an active L-band polarimetric radar sensor (PALSAR) whose highresolution data may be used for environmental and hazard monitoring. The third Polarimetric Spaceborne sensor is TerraSAR-X, a new German radar satellite, developed by DLR, EADS-Astrium and Infoterra GmbH, was launched on June 2007. This sensor carries a dual-polarimetric and high frequency X-Band SAR sensor that can be operated in different modes and offers features that were not available from space before. At least, the Polarimetric Spaceborne sensor, developed by CSA and MDA, and named RADARSAT-2 was launched in December 2007 The Radarsat program was born out the need for effective monitoring of Canada's icy waters, and some Radarsat- 2 capabilities that benefit sea- and river ice applications are the multi-polarization options that will improve ice-edge detection, ice-type discrimination and structure information. The many advances in these different Polarimetric Spaceborne platforms were developed to respond to specific needs for radar data in environmental monitoring applications around the world, like : sea- and river-ice monitoring, marine surveillance, disaster management, oil spill detection, snow monitoring, hydrology, mapping, geology, agriculture, soil characterisation, forestry applications (biomass, allometry, height...), urban mapping etc.... In order to promote the exploitation of Polarimetric Spaceborne data, as it is starting today to proliferate with the launch of these Polarimetric SAR sensors, the PolSARpro Software, developed under contract to ESA and that is a toolbox for the scientific exploitation of Polarimetric SAR and Polarimetric-Interferometric data and a tool for high-level education in radar polarimetry, has been expanded and refined to include all elements necessary for the demonstration of a number of key applications. The PolSARpro Software, that already was supporting an important range of airborne and spaceborne polarimetric data sources, supports now the following additional data sources: ALOS-PALSAR (Dual-Pol fine mode and Quad-Pol mode), TerraSAR-X (Dual-pol mode) and Radarsat-2 (Dual-Pol fine mode and Quad-Pol fine and standard modes), by offering a platform dedicated interface for E.O Scientific Investigator. A number of illustrations of key applications has been developed for the demonstration and the promotion of the Polarimetric Spaceborne missions, that are consistent with the activities incorporated in the GMES Services Element (GSE). The aim of this communication is to present the current state of the art in SAR Polarimetry ranging from theory to applications, with special emphasis in the analysis of data provided by the new Polarimetric Spaceborne SAR sensors, and samples of real polarimetric data will be presented for use in real-life examples of key applications.
\end{abstract}

This is an Open Access article distributed under the terms of the Creative Commons Attribution-Noncommercial License 3.0, which permits unrestricted use, distribution, and reproduction in any noncommercial medium, provided the original work is properly cited. 